\title{
Wolbachia infection influences the development of Culex pipiens embryo in incompatible crosses
}

\author{
O Duron and M Weill \\ Team Genetics of Adaptation, Laboratoire Génétique et Environnement, Institut des Sciences de l'Evolution (UMR CNRS 5554), \\ Université de Montpellier II (C.C. 065), F-34095 Montpellier cedex 05, France
}

\begin{abstract}
Wolbachia are maternally inherited endosymbiotic bacteria that infect many arthropod species and have evolved several different ways for manipulating their host, the most frequent being cytoplasmic incompatibility $(\mathrm{Cl})$. $\mathrm{Cl}$ leads to embryo death in crosses between infected males and uninfected females, as well as in crosses between individuals infected by incompatible Wolbachia strains. In the mosquito Culex pipiens, previous studies suggested developmental variation in embryos stemming from different incompatible crosses. We have investigated this variation in different incompatible crosses. Unhatched eggs were separated into three classes based upon the developmental stage reached by the embryos. We found that incompatible crosses involving uninfected females produced only embryos whose development was arrested at a very early stage, irrespective of the Wolbachia variant infecting the male. These results differ from other
\end{abstract}

host species where a developmental gradient that could reach late stages of embryogenesis or even living larvae was observed, and indicate a novel peculiarity of $\mathrm{Cl}$ mechanism in $C$. pipiens. By contrast, all incompatible crosses with infected C. pipiens females produced embryos of all three classes. The proportion of embryo classes appeared to be associated with the strains involved, suggesting specific $\mathrm{Cl}$ properties in different incompatible crosses. In addition, the contribution of parental genome was characterized in embryo classes using molecular markers for each chromosome. Embryo phenotypes appeared linked to the paternal chromosomes' contribution, as described in Drosophila simulans. However, this contribution varied according to maternal infection and independently of male factors.

Heredity (2006) 96, 493-500. doi:10.1038/sj.hdy.6800831; published online 26 April 2006

Keywords: Wolbachia; Culex pipiens; cytoplasmic incompatibility; embryo development

\section{Introduction}

Wolbachia is a genus of maternally inherited bacteria, widespread among arthropods and filarial nematodes. The successful spread of Wolbachia in arthropods is attributed to their ability to alter host reproduction to their own advantage (Rousset and Raymond, 1991), including feminization, male-killing, parthenogenesis and most commonly cytoplasmic incompatibility (CI) (Werren, 1997; Stouthamer et al, 1999). CI results from inappropriate interactions between sperm and egg, leading generally to embryonic mortality or to the production of male excess in some haplodiploid species. CI occurs when infected males mate either with uninfected females or with females infected by incompatible Wolbachia strains. CI has been usually interpreted as resulting from two bacterial components: a mod function (for modification) that would affect sperm and induce embryo death, and a resc function (for rescue) provided by the Wolbachia present in the egg that would restore male and female chromosomes coordination in compatible crosses (Werren, 1997). In CI embryos (ie embryos from an incompatible cross), the paternal chromosomes

Correspondence: $M$ Weill, Team Genetics of Adaptation, Laboratoire Génétique et Environnement, Institut des Sciences de l'Evolution (UMR CNRS 5554), Université de Montpellier II (C.C. 065), F-34095 Montpellier cedex 05, France. E-mail: weill@isem.univ-montp2.fr Received 13 September 2005; accepted 6 March 2006; published online 26 April 2006 are improperly condensed, and do not accurately segregate during the first zygotic mitosis, while the maternal chromosomes condense and segregate normally. This results in embryos with a complete set of maternal chromosomes, but a reduction or absence of paternal chromosomes (reviewed in Tram et al, 2003). CI embryos from mosquitoes, flies and wasps exhibit the same defects, suggesting a conservative mechanism induced by Wolbachia (Tram et al, 2003).

Three main phenotypic classes were observed in eggs stemming from incompatible crosses involving uninfected females (in Aedes polynesiensis and Drosophila simulans) as well as infected females (in D. simulans) (see Wright and Barr (1981) for Aedes data, and Callaini et al (1996) for Drosophila data). The first class is represented by eggs with no detectable embryogenesis, while the two other classes correspond to embryos reaching medium or late developmental stages. The first class of incompatible embryos appeared to be aneuploid, with a complete set of maternal chromosomes but a reduced number of paternal chromosomes. Embryos of the second and third classes were haploid, with maternal but no paternal chromosomes (Callaini et al, 1996). However, the severity of the chromosome defects does not influence the final CI phenotype, as both aneuploid and haploid embryos die.

Among all the studied host species, mosquitoes of the Culex pipiens complex exhibit the highest variability, both of CI crossing types (Laven, 1967; Guillemaud et al, 1997) and of Wolbachia polymorphism, detected using rapidly 
evolving markers (Duron et al, 2005, 2006). Very little is known about embryo differentiation in Culex CI crosses, and authors using different mosquito strains have reported contradictory results. In CI embryos, the haploid female pronucleus has been reported to divide, and to develop up to histological differentiation in the majority of eggs (Jost, 1970a, b, 1971). The distinction between incompatible and unfertilized egg rafts has thus generally been made on the basis of embryo development phenotype (Jost, 1970b). More recently, Rasgon and Scott (2003) did not observe evidence of embryo development in fertilized incompatible eggs and concluded that absence of embryo development was inadequate to differentiate incompatible and unfertilized eggs. This discrepancy suggests a phenotypic variation of CI embryos, and the factors involved in this polymorphism need to be clarified.

We have here investigated embryo development in eggs produced by incompatible crosses between infected males and uninfected females or females infected by incompatible Wolbachia strains. Data for embryo development stages, hatching rate and genetic characteristics were collected, and compared between crosses. Genetic markers specific to paternal or maternal mosquito chromosomes were developed to characterize the ploidy status of CI embryos. Our results disclose a novel peculiarity of $\mathrm{CI}$ in $\mathrm{C}$. pipiens: all incompatible crosses involving uninfected females produced eggs in which embryonic development was arrested at very early stages, irrespective of the Wolbachia variant infecting the males. Moreover, the contribution of paternal chromosomes in CI embryos was reduced when mothers were infected, independently of male components.

\section{Materials and methods}

\section{Mosquito strains}

Four laboratory strains of the C. pipiens complex, differing by their geographical origin and Wolbachia genotype, were used. Two strains belong to the C. $p$. quinquefasciatus form: Slab (Georghiou et al, 1966) and MaClo (Duron et al, 2006), originally collected in California in 1954 and 1984, respectively. The two other strains belong to the C. p. molestus form: Istanbul (Duron et al, 2005) and Tunis (Ben Cheikh et al, 1998), collected in Turkey (2003) and Tunisia (1992), respectively. Istanbul and Tunis were both infected by wPip3, MaClo by wPip4 and Slab by wPip1/4 Wolbachia variants characterized by the $\operatorname{Tr} 1$ transposable element (Duron et al, 2005). These strains were found to be genetically different and monoinfected using WO prophage markers (Duron et al, 2006).

Strains free of Wolbachia were generated by a modification of the technique described by Portaro and Barr (1975). Larvae were reared for three generations in a solution containing the antibiotic tetracycline hydrochloride at $10^{-4}, 2 \times 10^{-4}$ and $4 \times 10^{-4} \mathrm{M}$ for the first, second and third generations, respectively. Loss of Wolbachia was controlled by PCR amplification of a fragment of the wsp gene using the specific primers wolpipdir and wolpiprev described by Berticat et al (2002). Mosquito DNA was extracted using a CTAB protocol (Rogers and Bendich, 1988). Quality of DNA was evaluated using PCR amplification of the Culex acetylcholinesterase ace-2 gene with primers described by Weill et al (2000). Wolbachia-free strains are referred as TC-treated. Thus, SlabTC, MaCloTC, TunisTC and IstanbulTC are uninfected strains derived from Slab, MaClo, Tunis and Istanbul, respectively. To insure that differences of hatching rate were not due to antibiotic toxicity, TC strains were reared for at least four generations in standard laboratory conditions, without tetracycline, before the beginning of crossing experiments.

\section{Crossing experiments}

Reciprocal mass crosses between 25 males and females reared in controlled conditions were used for each pair of strains. Two-day old adults were used in order to avoid the effect of age on fecundity. After 6 days, females were blood-fed. Egg-rafts (between 50 and 300 eggs per raft) were collected daily. Each cross was characterized by (i) the total number of eggs; (ii) the number of egg-rafts; (iii) the mean proportion of developed embryos and (iv) the mean proportion of hatched eggs, which indicates the CI level. The proportion of embryos and hatching rate were determined using a binocular magnifying loupe. When an egg-raft did not produce any larva, the spermathacae of the corresponding female was checked for insemination. Egg-rafts from noninseminated females were discarded. The following types of egg were thus obtained:

(1) unfertilized eggs, by blood feeding infected and uninfected females reared in absence of males;

(2) compatible eggs, by crossing infected and uninfected mosquitoes of the same strains;

(3) incompatible eggs, by crossing uninfected females with infected males, and incompatible infected mosquitoes of different strains. Incompatible crosses were repeated twice for validation. These incompatible crosses were also made using uninfected males to assess the implication of Wolbachia in the observed incompatibilities.

When incompatible crosses produced occasional larvae, they were reared until the imaginal stage for analysis.

\section{Embryo observations}

Egg-rafts from each cross were collected $36 \mathrm{~h}$ after oviposition (approximately less than $8 \mathrm{~h}$ before hatching), and divided into two groups. The first group was maintained in standard conditions for hatching. Egg-rafts from the second group were broken, and individualized eggs were observed using binocular magnifying loupe. Photographs were taken using with a Kyocera Finecam S5.

\section{Contribution of parental chromosomes}

Parental chromosomes were studied in embryos (or eventually larvae and adults) from crosses using different $C$. pipiens subspecies, that is, $q \mathrm{MaClo} \times \delta$ Istanbul (or $\hat{o}$ IstanbulTC) and $q \mathrm{Slab} \times \hat{\sigma}$ Tunis crosses.

The C. pipiens karyotype is represented by three pairs of homomorphic chromosomes (Clements, 1992). Each parental chromosome was characterized by a specific molecular PCR/RFLP diagnosis marker from the ace-2, ace-1 or $k d r$ genes. 
The first gene, ace-2, is located on chromosome I and codes for the acetylcholinesterase 2 (AChE2) (Malcolm et al, 1998). A PCR/RFLP test on ace-2 gene was previously reported to discriminate between quinquefasciatus (MaClo and Slab strains) and pipiens/molestus (Istanbul and Tunis strains) subspecies (Bourguet et al, 1998) (Figure 1).

The second gene, ace-1, is located on chromosome II and codes for the synaptic AChE1 (Weill et al, 2003). A $526 \mathrm{bp}$ ace-1 fragment was amplified using primers CxEx3dir (5'-CGA CTC GGA CCC ACT CGT) and CxEx3rev (5'-GTT CTG ATC AAA CAG CCC CGC). Sequencing was performed directly on PCR products using an ABI prism 310 sequencer using the Big Dye Terminator kit. A BsrBI digestion of the PCR product allowed discrimination between Slab and MaClo from Istanbul and Tunis. BsrBI cuts Slab and MaClo ace-1 fragments twice (positions +135 and +276 ) and the Istanbul and Tunis ace-1 fragments only once $(+135)$ (Figure 1).

The third gene, $k d r$, is located on chromosome III and codes for a voltage-dependent sodium channel (Halliday and Georghiou, 1985). A $k d r$ gene fragment was amplified using Cgd1 and Cgd2 primers (MartinezTorres et al, 1999) producing fragments of $500 \mathrm{bp}$ (Slab), $492 \mathrm{bp}$ (MaClo), $516 \mathrm{bp}$ (Tunis) and $504 \mathrm{bp}$ (Istanbul), due to variable size of introns located within the $k d r$ gene (Martinez-Torres et al, 1999). The RsaI enzyme cuts Slab, $\mathrm{MaClo}$, and Istanbul $k d r$ fragment once (positions +256 , +252 and +360 , respectively) and the Tunis $k d r$ fragment twice $(+154$ and +371$)$ (Figure 1).

Each marker represents only a small part of its corresponding chromosome. The diagnosis thus does not allow the detection of partial chromosome deletions.

\section{Statistical analysis}

Results were analysed by means of generalized linear models (GLM) and Mann-Whitney tests. We analysed proportion of developed embryos (EMB) and hatching rate (HR) in egg-rafts from incompatible crosses with the GLM. Each egg-raft was characterized by four variables: the proportion of developed embryos (EMB), hatching rate (HR), maternal strain (FEM: six levels) and paternal strain (MAL: four levels). For both dependant variables EMB and HR, the linear model FEM $\times$ MAL was fitted. This model was then simplified according to Crawley (1993). Normality of residuals from the minimal model was tested using Lilliefors test (Dallal and Wilkinson,
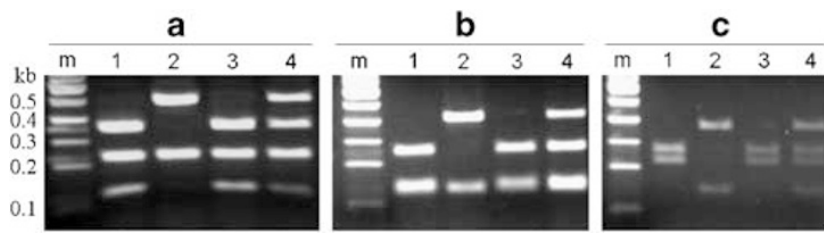

Figure 1 Restriction profiles of chromosomes markers. Restriction profiles of the PCR products of (a) ace-2 gene by ScaI enzyme, (b) ace-1 by $B s r B I$ and (c) $k d r$ by RsaI from single mosquito extracted DNA. m: molecular weight marker; 1 : MaClo strain; 2: Istanbul strain; 3: third class embryo issued from incompatible cross between $+\mathrm{MaClo} \times{ }_{0}$ Istanbul (embryo displayed only maternal markers); 4 : viable embryo from compatible cross between $q \mathrm{MaClo} \times \delta$ IstanbulTC (embryo displayed both maternal and paternal markers).
1986). Calculations were performed using the $R$ free software (R Development Core Team, 2004).

\section{Results}

\section{Compatibility status}

Females from infected and uninfected strains were able to lay few eggs without fertilization, and as expected no hatching was recorded among the eggs of the 35 females (Table 1).

No incompatibility was detected in crosses between mosquitoes belonging to the same strain (infected or uninfected), all hatching rates being in the $83-98 \%$ interval (Table 1 and Figure 2).

Uninfected females were crossed with (i) infected males from the strains they derived ( $q$ SlabTC $\times$ o Slab o $\mathrm{MaCloTC} \times$ o $\mathrm{MaClo} ;$ o IstanbulTC $\times \widehat{\delta}$ Istanbul) and (ii) infected males from other strains ( $q$ SlabTC $\times \delta$ Tunis and $\hat{o}$ MaClo; $q$ MaCloTC $\times \hat{o}$ Istanbul; $q$ IstanbulTC $\times \delta$ Slab). Complete CI occurred when infected males (irrespective of their strain origin) mated with uninfected females (no hatching; Table 1 and Figure 2).

Four incompatible crosses between infected strains were studied, all producing few or no larvae $(q$ Slab $\times \widehat{\delta}$ Tunis and $\hat{o}$ MaClo; $q$ MaClo $\times \hat{o}$ Istanbul; $q$ Istanbul $\times$ o Slab; Table 1). Not all possible crosses were tested, and more CI may occur between these strains.

In $q$ Slab $\times \hat{0}$ Tunis crosses, a $1.1 \%$ hatching rate was observed, and all larvae $(n=19$; Table 1$)$ were reared. Six reached the adult stage: $5 q$ and 10 . The remaining larvae $(n=13)$ reached the second instar stage and survived up to 3 weeks. All other incompatible crosses produced no larvae. Hatching rate in incompatible egg-rafts was not related to female strain $\left(F_{1,150}=0.14\right.$, $P=0.71)$ or to male strain $\left(F_{1,150}=0.13, P=0.16\right)$, that is, embryo mortality in incompatible crosses did not differ according to the parental strains.

Incompatible crosses were repeated using uninfected males to test Wolbachia involvement. Crosses between any TC males and infected females $(q$ Slab $\times \hat{\sigma}$ TunisTC

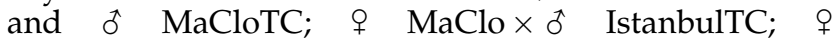
Istanbul $\times$ o SlabTC) displayed hatching rates within the 92-98\% interval (Table 1 and Figure 2). Elimination of male Wolbachia completely restored the compatibility of these crosses. Thus, Wolbachia infection was responsible for all the incompatibility observed.

\section{Embryos development}

In compatible crosses, normal embryos were observed $36 \mathrm{~h}$ after oviposition in 85-99\% of eggs (the remaining eggs did not show evidence of embryo presence) (Table 1 and Figure 2). Their development followed Clements' description (1992): head capsule with mouthparts, thorax and segmented abdomen were observed (Figure 3f). No difference was observed between infected and uninfected compatible embryos.

By contrast, the number of viable embryos in incompatible crosses was severely reduced as mortality was above 98\% (Table 1 and Figure 2). However, developed embryos were observed in some incompatible egg-rafts and their frequency was correlated with parental strain $\left(F_{1,148}=85.01, P<10^{-15}\right)$. No embryonic development was observed among the large majority ( $>99 \%$ ) of eggs 
Table 1 Incompatibility relationships between infected and uninfected (TC) strains

\begin{tabular}{|c|c|c|c|c|c|}
\hline \multicolumn{2}{|c|}{ Cross } & \multirow{2}{*}{$\frac{\text { Number of eggs }}{663}$} & \multirow{2}{*}{$\frac{\text { Number of egg-rafts }}{14}$} & \multirow{2}{*}{$\begin{array}{c}\text { Proportion of developed embryos } \\
0.000 \pm 0.000\end{array}$} & \multirow{2}{*}{$\frac{\text { Hatching rate }}{0.000 \pm 0.000}$} \\
\hline q Slab & - & & & & \\
\hline ㅇ Slab & ô Slab & 2131 & 14 & $0.980 \pm 0.022$ & $0.977 \pm 0.023$ \\
\hline q Slab & $\widehat{o}$ Tunis & 1835 & 14 & $0.193 \pm 0.117$ & $0.011 \pm 0.024$ \\
\hline q Slab & o TunisTC & 957 & 8 & $0.985 \pm 0.017$ & $0.962 \pm 0.042$ \\
\hline o Slab & o MaClo & 1628 & 15 & $0.530 \pm 0.235$ & $0.000 \pm 0.000$ \\
\hline i Slab & đิ MaCloTC & 2595 & 20 & $0.955 \pm 0.039$ & $0.942 \pm 0.043$ \\
\hline \& SlabTC & - & 209 & 3 & $0.000 \pm 0.000$ & $0.000 \pm 0.000$ \\
\hline q SlabTC & ô Slab & 2735 & 16 & $0.000 \pm 0.000$ & $0.000 \pm 0.000$ \\
\hline \& SlabTC & o SlabTC & 1881 & 12 & $0.985 \pm 0.012$ & $0.982 \pm 0.010$ \\
\hline q SlabTC & $\widehat{0}$ Tunis & 3612 & 22 & $0.000 \pm 0.000$ & $0.000 \pm 0.000$ \\
\hline o SlabTC & ơ MaClo & 2640 & 18 & $0.000 \pm 0.000$ & $0.000 \pm 0.000$ \\
\hline ㅇ MaClo & - & 171 & 2 & $0.000 \pm 0.000$ & $0.000 \pm 0.000$ \\
\hline ¡ MaClo & o MaClo & 1190 & 9 & $0.934 \pm 0.057$ & $0.920 \pm 0.053$ \\
\hline \& MaClo & $\delta$ Istanbul & 2333 & 17 & $0.695 \pm 0.189$ & $0.000 \pm 0.000$ \\
\hline q MaClo & ô IstanbulTC & 2129 & 14 & $0.959 \pm 0.025$ & $0.910 \pm 0.049$ \\
\hline क MaCloTC & - & 345 & 3 & $0.000 \pm 0.000$ & $0.000 \pm 0.000$ \\
\hline \& MaCloTC & ô MaClo & 1547 & 12 & $0.000 \pm 0.000$ & $0.000 \pm 0.000$ \\
\hline o MaCloTC & o MaCloTC & 1343 & 10 & $0.964 \pm 0.028$ & $0.934 \pm 0.029$ \\
\hline q MaCloTC & ô Istanbul & 1893 & 15 & $0.000 \pm 0.000$ & $0.000 \pm 0.000$ \\
\hline ㅇ Istanbul & - & 518 & 8 & $0.000 \pm 0.000$ & $0.000 \pm 0.000$ \\
\hline ᄋ Istanbul & ô Istanbul & 1313 & 10 & $0.978 \pm 0.019$ & $0.974 \pm 0.021$ \\
\hline ㅇ Istanbul & ô Slab & 1697 & 16 & $0.576 \pm 0.261$ & $0.000 \pm 0.000$ \\
\hline q Istanbul & ò SlabTC & 1486 & 11 & $0.986 \pm 0.014$ & $0.980 \pm 0.014$ \\
\hline ㅇ IstanbulTC & - & 355 & 5 & $0.000 \pm 0.000$ & $0.000 \pm 0.000$ \\
\hline † IstanbulTC & $\hat{o}$ Istanbul & 169 & 2 & $0.026 \pm 0.011$ & $0.000 \pm 0.000$ \\
\hline † IstanbulTC & ô IstanbulTC & 1014 & 8 & $0.858 \pm 0.087$ & $0.837 \pm 0.071$ \\
\hline o IstanbulTC & ô Slab & 783 & 7 & $0.008 \pm 0.011$ & $0.000 \pm 0.000$ \\
\hline
\end{tabular}

Developed embryos refer to second and third class embryos.
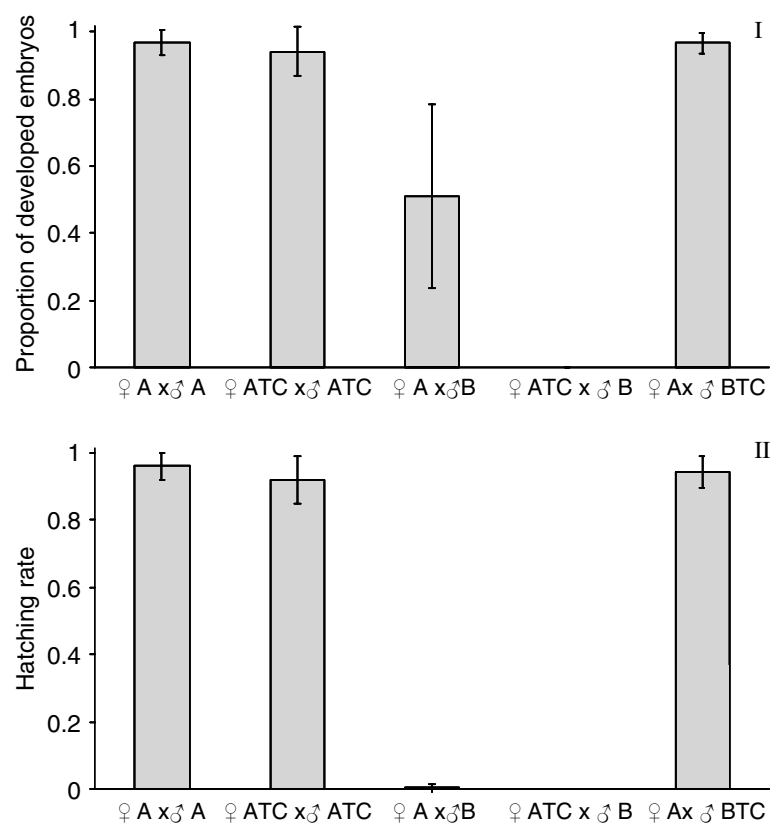

Figure 2 (I) Mean proportions of developed embryos (representing second and third class embryos) and (II) mean of hatching rate in compatible and incompatible crosses. Strains were pooled in A and $\mathrm{B}$ groups according to their crossing type. A and B represent infected and incompatible mosquito groups. TC strains correspond to uninfected strains. $q \mathrm{~A} \times \sigma^{\pi} \mathrm{A}, q \mathrm{ATC} \times \widehat{0} \mathrm{ATC}$ and $q \mathrm{~A} \times \widehat{0}$ $\mathrm{BTC}$ are compatible crosses; $+\mathrm{A} \times \hat{\delta} \mathrm{B}$ and $q \mathrm{ATC} \times \hat{\delta} \mathrm{B}$ are incompatible crosses. issued from incompatible crosses between uninfected females and all infected males tested. These eggs (Figure 3b) were similar to unfertilized eggs (Figure 3a), in which no mitotic events have occurred. They harboured a dense mass immersed in a less compact fluid that was frequently fragmented in smaller parts, and showed no evidence of cellular organization. In incompatible crosses involving $q$ IstanbulTC, a few eggs $(<1 \%)$ containing an embryo with stemmata (ie proeyes) were very occasionally observed (Table 1).

Eggs from incompatible crosses between infected mosquitoes were divided into three classes according to the phenotype exhibited $36 \mathrm{~h}$ after oviposition (Figure 3) (Callaini et al, 1996). Class 1 eggs (Figure 3c) were phenotypically similar to unfertilized eggs or eggs produced by uninfected females crossed with infected males (Figure 3a and b). The second class (Figure 3d) was characterized by a more homogeneous content and had organized tissues, as judged by the presence of stemmata, but embryos failed to differentiate properly. More than two stemmata were frequently observed, often in a medial position, contrarily to compatible embryos (Figure 3f) that harbour anterior stemmata. The third class (Figure 3e) was composed of eggs with embryos whose development had stopped shortly before hatching. These embryos had a differentiated head with maxillary appendages, thorax and segmented abdomen. Some displayed muscular contractions as observed in compatible embryos. However, they showed severe signs of disorganization. The most prominent abnormality was 


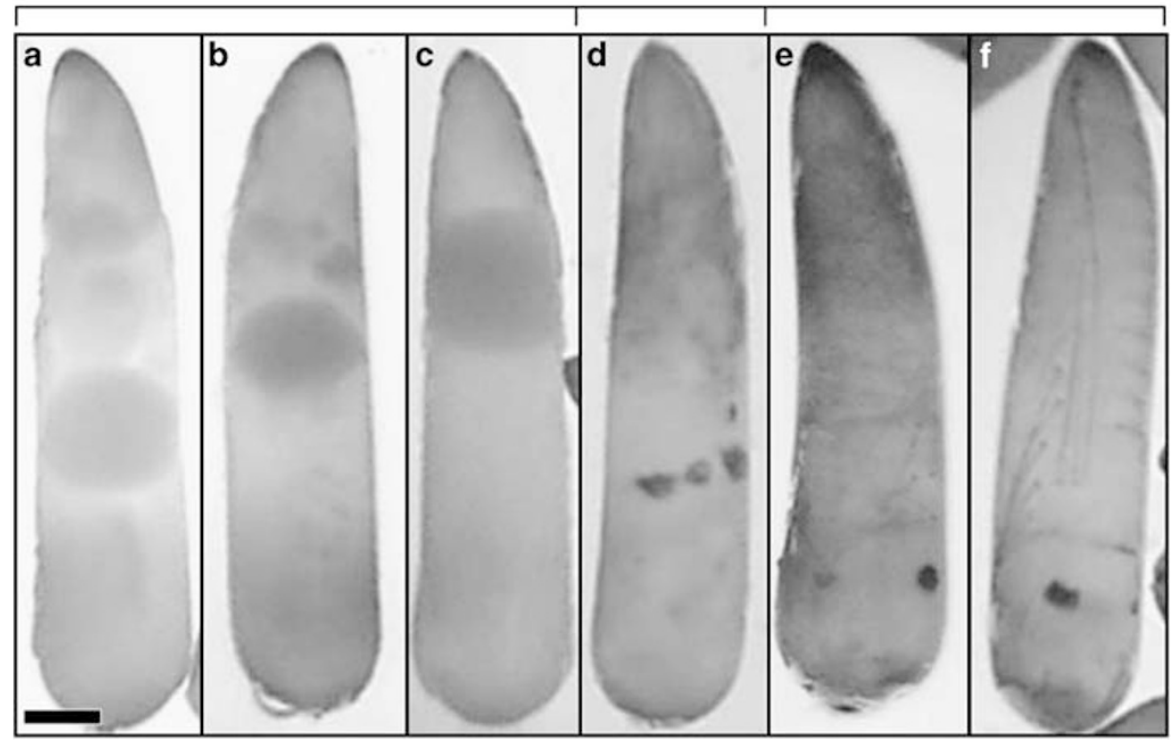

Figure 3 Culex pipiens eggs $36 \mathrm{~h}$ after oviposition. I, II and III refer to first, second and third class embryos, respectively. (a) Unfertilized egg, its phenotype is characterized by a central dense mass (first class embryo). (b) First class embryo from incompatible cross $\$$ MaCloTC $\times \hat{\delta}$ Istanbul, the egg is identical to unfertilized egg (a). (c) First class embryo from $q$ MaClo $\times \delta$ Istanbul incompatible cross, the phenotype is similar to (a) and (b). (d) Second class embryo from $q \mathrm{MaClo} \times \delta$ Istanbul incompatible cross, three stemmata are present, shifted in a medium position and irregularly outlined. (e) Third class embryo from $q$ MaClo $\times \hat{\delta}$ Istanbul incompatible cross, the embryo has reached the highest degree of development, but shows irregular orientation of bristles. (f) Embryo from the compatible $q$ MaClo $\times \delta^{\tau}$ IstanbulTC cross; note head capsule with mouthparts, thorax and segmented abdomen. Bar: $50 \mu \mathrm{m}$.

an irregular orientation of bristles, which were often tangled, whereas in compatible embryos, bristles were regularly organized and perfectly parallel. Some embryos had no apparent abnormalities but did not hatch, and died within the egg. After 2-3 days, the cytoplasm of the embryos from the first two classes remained white, while the cytoplasm of embryos from the third class gradually became brown. All occasional larvae $(n=19)$ issued from the $O$ Slab $\times \widehat{0}$ Tunis cross showed normal physical organization, and no evidence of developmental aberrations.

The second and third classes were pooled for counting. Their frequencies varied depending on the strains crossed (19.3\% in + Slab $\times$ o Tunis; $53.0 \%$ in + Slab $\times \widehat{0}$ MaClo; $69.5 \%$ in $q$ MaClo $\times \delta$ Istanbul; $57.6 \%$ in $q$ Istanbul $\times$ o Slab; Table 1, Figures 2 and 4). These frequencies were significantly different (Mann-Whitney two-sided test; $P<0.01$ ), except for $q \mathrm{Slab} \times \delta \mathrm{MaClo}$ and $q$ Istanbul $\times$ o Slab crosses comparisons $(P>0.30)$.

\section{Parental chromosomes characterization}

Chromosomes origin was determined for 16-32 single embryos from 4 to 8 egg-rafts issued from (i) compatible crosses between infected females and uninfected males; (ii) incompatible crosses between uninfected females and infected males and (iii) incompatible crosses between infected females and males.

All embryos $(n=16)$ from the $q \mathrm{MaClo} \times \delta$ IstanbulTC compatible cross displayed both maternal and paternal markers for each of the three chromosome (Table 2), indicating that embryos were diploid with an equal contribution of paternal and maternal genetic material.

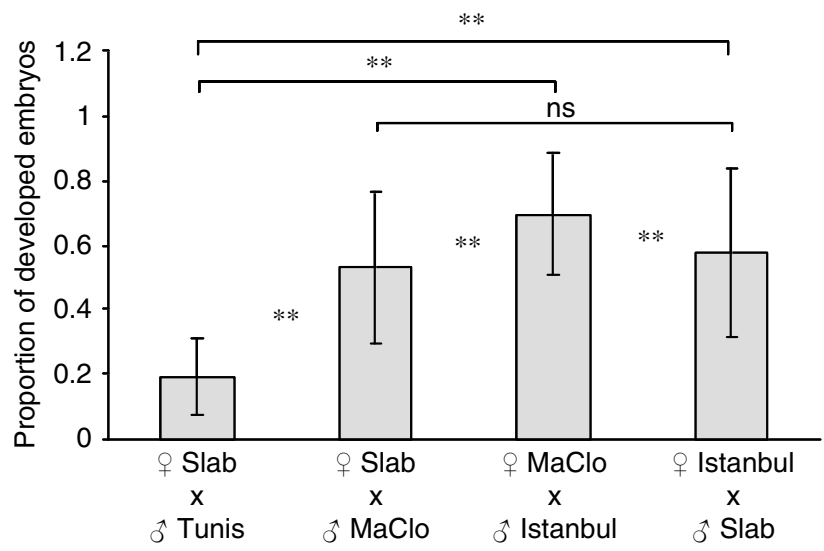

Figure 4 Mean proportions of developed embryos (second and third class) in incompatible crosses between infected strains. All pair comparisons were significantly different except for $q$ Slab $\times \delta$ $\mathrm{MaClo}$ and $q$ Istanbul $\times \hat{\delta}$ Slab crosses. ns, nonsignificant $P$-value; ${ }^{* *} P<0.01$

Individual eggs $(n=32)$ from the $q$ MaCloTC $\times \delta$ Istanbul incompatible cross (class 1 embryos) were not analysed as insufficient DNA could be extracted. PCR carried out using DNA extracted from complete egg-rafts $(n=7)$ still failed for some loci. In the case where amplification was efficient, egg-rafts displayed maternal and paternal markers for each chromosome (Table 2).

In the $q \mathrm{MaClo} \times \widehat{\sigma}$ Istanbul incompatible cross, class 1 embryos $(n=16)$ could not be analysed for the same reason. Eggs in the second and the third classes contained embryos $(n=16)$ with maternal but no paternal markers at each locus (Table 2). 
Table 2 Chromosomes characterization of embryos, larvae and adults issued from both compatible and incompatible crosses

\begin{tabular}{|c|c|c|c|c|}
\hline Cross & $\mathrm{n}$ and stage tested & Ch I (ace-2 gene) & Ch II (ace-1 gene) & Ch III ( $\mathrm{kdr}$ gene) \\
\hline o $\mathrm{MaClo} \times \widehat{o}$ IstanbulTC (compatible) & 16 embryos & $m / p(16)$ & $m / p(16)$ & $m / p(16)$ \\
\hline o $\mathrm{MaClo} \times \hat{o}$ Istanbul (incompatible) & 32 first class embryos & - & - & - \\
\hline o $\mathrm{MaClo} \times \widehat{o}$ Istanbul (incompatible) & 16 second and third class embryos & $m(16)$ & $m(16)$ & $m(16)$ \\
\hline o MaCloTC $\times$ ơ Istanbul (incompatible) & Seven egg-rafts (first class embryos) & $m / p(1)^{\mathrm{a}}$ & $m / p(7)$ & $m / p(4)^{\mathrm{a}}$ \\
\hline o Slab $\times$ o Tunis (incompatible) & 16 first class embryos & - & - & - \\
\hline ㅇ Slab $\times$ ơ Tunis (incompatible) & 16 second and third class & $\begin{array}{c}m(10) \\
m / p(6)\end{array}$ & $\begin{array}{c}m(10) \\
m / p(6)\end{array}$ & $\begin{array}{c}m(10) \\
m / p(6)\end{array}$ \\
\hline o Slab $\times$ o Tunis (incompatible) & 6 adults and 13 larvae & $m / p(19)$ & $m / p(19)$ & $m / p(19)$ \\
\hline
\end{tabular}

Ch refers to chromosome, corresponding gene being indicated. $m$ and $p$ correspond to maternal and paternal markers, respectively. $m / p$ individuals are heterozygous, whereas $m$ individuals express only maternal markers. Number of each observation is in parenthesis. No PCR products were obtained for individual first class embryos, preventing chromosome analysis.

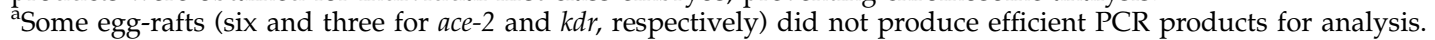

Table 3 Phenotypic class composition according to host species in crosses between infected males and uninfected females

\begin{tabular}{lllll}
\hline Host species & Wolbachia variant & Embryo phenotypes & Hatching rate (\%) & References \\
\hline Culex pipiens & $w$ Pip3, 4 and 1/4 & First class & 0 & This study \\
Aedes polynesiensis & wScu & First, second and third classes & $7-10$ & Wright and Barr (1981) \\
A. albopictus & $w \mathrm{AlbA}$ & Undocumented & 0 & Otsuka and Takaoka (1997) \\
A. albopictus & $w \mathrm{AlbB}$ & Undocumented & 0 & Xi et al (2005) \\
Drosophila simulans & $w \mathrm{Ri}$ & First, second and third classes & $0-10$ & Callaini et al (1996) \\
D. simulans & $w \mathrm{Ha}$ & Undocumented & $0-50$ & Merçot and Charlat (2003) \\
D. simulans & $w \mathrm{No}$ & Undocumented & $20-60$ & Merçot and Charlat (2003) \\
D. melanogaster & $w \mathrm{Mel}$ & Undocumented & $0-80$ & Merçot and Charlat (2003) \\
\hline
\end{tabular}

Host listing is not exhaustive.

In the o Slab $\times$ o Tunis incompatible cross, no PCR amplification was obtained from the class 1 embryos $(n=16)$. Among the 16 embryos from eggs in the second and third classes, 10 displayed maternal but not paternal markers at each locus, and six had both parental markers (Table 2). As the cross o Slab $\times \hat{0}$ Tunis provides some larvae, it is likely that embryos with complete maternal and paternal chromosome set were viable and able to hatch (Table 1). This interpretation is supported by the analysis of the 19 hatched larvae from this cross, 13 of which died during their preimaginal development, of which six reached the adult stage $(5 \quad q$ and $1 \quad \hat{\sigma})$. All displayed both maternal and paternal markers (Table 2).

\section{Discussion}

Our data agree with previous findings reporting a high frequency of embryonic death in C. pipiens incompatible crosses (Laven, 1967; Guillemaud et al, 1997). However, we have shown that embryonic development varied considerably, depending on the female infection status and the cross type involved.

\section{Phenotypes distribution of $\mathrm{Cl}$ embryos}

By examining eggs obtained from incompatible crosses, three main categories of development $36 \mathrm{~h}$ after oviposition have been distinguished. These three classes were present in incompatible crosses involving infected females, although their proportion varied considerably with the maternal strain. In contrast, when females were uninfected, there was no evidence of cell division (class 1 eggs) in any CI eggs. These observations concerned crosses involving three uninfected female strains, and four infected male strains, representing three Wolbachia variants (wPip3, wPip4 and wPip1/4).
Our results using C. pipiens contrast with observations in other insect species in respect to the homogeneity of CI embryo phenotypes produced by uninfected females (Table 3). In D. simulans, the three embryo classes were observed in all incompatible cross types, whether females were infected or not (Callaini et al, 1996). Moreover, in D. simulans and D. melanogaster, complete $\mathrm{CI}$ in crosses involving uninfected females is rare and living larvae are frequently observed (Merçot and Charlat, 2003). Similarly, in A. polynesiensis, a gradient of embryo development was observed in incompatible crosses between infected males and uninfected females (Wright and Barr, 1981).

In C. pipiens, a fertilized incompatible egg can be identified from an observation of embryo development in crosses between infected mosquitoes, but not in crosses in which females are uninfected. Thus, the observation of embryo development in C. pipiens eggs from a cross showing $\mathrm{CI}$ constitutes strong evidence that the mother was infected as stated by Jost (1970a, b, 1971): see Table 3. Rasgon and Scott (2003) did not observe evidence of embryo development in eggs produced by fertilized females; this result can be explained because mothers were uninfected in their crosses.

The difference observed between uninfected and infected females indicates that maternal Wolbachia present in the eggs allow some morphogenesis. However, Wolbachia are not necessary for embryogenesis since embryos from uninfected females develop correctly when males are not infected.

Interestingly, the proportion of different embryo classes varied among infected strains. This variation implicates male factors in embryos' capacity to reach advanced stages. In incompatible crosses involving $q$ Slab, $53.0 \%$ of eggs contained developed embryos (ie from second and third classes) when fertilized by $\delta$ 
MaClo, whereas only $19.3 \%$ were present when fertilized by $\delta$ Tunis (Mann-Whitney two-sided test; $P<10^{-3}$ ) (Figure 4).

These results suggest that, in C. pipiens, each incompatible strains combination involves peculiar Wolbachia interactions and thus CI properties. The CI mechanism in C. pipiens would appear more complex than expected, with mechanisms specific to each strain combination. Further investigations of this phenomenon are needed given the frequency and variety of incompatibilities (Laven, 1967; Guillemaud et al, 1997): Wolbachia infection appears to be nearly universal in natural populations (Duron et al, 2005), and more than 60 Wolbachia variants have been described (Duron et al, 2006).

\section{Chromosomes origin of embryo phenotypes}

An explanation for the different phenotypes found in CI embryos from $C$. pipiens could be the varying loss of paternal genetic material, which can induce haploidy or aneuploidy. Our investigation of chromosomes markers showed that, as expected, compatible embryos carried a complete set of both maternal and paternal chromosomes, allowing their correct development.

The paternal chromosomes contribution in the class 1 CI embryos remains unclear because no DNA could be obtained from single eggs. DNA extracted from whole egg-rafts, containing only class 1 embryos, revealed maternal and paternal markers from each chromosome. Two nonexclusive hypotheses may explain this observation: (i) embryos were diploid or (ii) embryos were aneuploid, each with a complete set of maternal chromosomes but only one or two paternal chromosomes. A complete set of paternal markers would then have been detected in whole egg-rafts because different chromosomes were present in different aneuploid embryos. It can be noted that class 1 embryos in $D$. simulans were found to be aneuploid, and that Tram et al (2003) suggested that it was a general phenomenon in incompatible crosses. Our analysis cannot exclude this possibility.

Incompatible embryos of the second and third classes had no paternal chromosomes, and were either haploid or diploid for the maternal set as described by Jost (1971) and Raymond et al (1986). Their failure to develop normally may be due to recessive lethal factors. $D$. simulans second and third classes embryos were also found to be haploid and inviable (Callaini et al, 1996; Lassy and Karr, 1996). Clearly, these embryos do not need a male genetic contribution to enter in division, but their complete cell differentiation requires some sperm factors (ie sperm penetration and/or extranuclear sperm components) since unfertilized eggs do not show embryo development.

A hypothesis to explain the variable loss of paternal chromosomes is based on the intensity of sperm modification by Wolbachia (see Tram et al, 2003). Since Wolbachia are present in testes but are absent from mature sperm, it has been proposed that Wolbachia induce CI by modifying sperm (Bressac and Rousset, 1993). If sperm modification is complete (ie all paternal chromosomes are efficiently modified by Wolbachia), a complete paternal genome loss will result in CI embryo. By contrast, if Wolbachia modification of paternal chromosomes is less severe, abnormally processed paternal chromosomes will segregate and a few will be transferred in CI embryo producing a lethal aneuploid genotype. This hypothesis implies that sperm modification, and thus paternal chromosomes contribution, will depend on male factors (including the Wolbachia variant or nuclear restorer). In this study of C. pipiens, we showed that the karyotype of CI embryos, that is, the contribution of paternal chromosomes, varies between uninfected and infected mothers and independently of male factors. For example, when males of the Istanbul strain were crossed with uninfected MaCloTC females, all embryos were aneuploid or diploid (cf above paragraph), whereas high frequency of haploid embryos was observed when the same males were crossed with infected MaClo females. This observation indicates that maternal Wolbachia present into Culex egg prevent the incorporation of the paternal genome into the embryo. The underlying mechanism remains to be identified.

Diploid parthenogenetic embryos able to hatch were described in $0.1 \%$ of $C$. pipiens embryos obtained from CI crosses, whereas all remaining embryos died (Jost, 1970a). They have been explained as the result of fusion between the products of the mother's meiosis, that is, fusion of the female pronucleus with a polar body or fusion of two polar bodies (Jost, 1970a, b, 1971). Raymond et al (1986) also observed occasional larvae from incompatible crosses, but some males were obtained and only few females were parthenogenetic. In this study, all viable larvae obtained from incompatible crosses displayed both maternal and paternal chromosomes, rejecting the possibility of efficient parthenogenesis in our crosses. The capacity for induction of parthenogenesis seems, then, to depend on the strains involved. Heterogeneity of Wolbachia and/or host factors could explain this polymorphism, suggesting that specific CI mechanisms could be invoked in some incompatible crosses.

A few viable CI larvae were obtained from the $q$ Slab $\times$ of Tunis cross, all with a complete set of maternal and paternal chromosomes. Their occurrence may be due to (i) occasional male nonmodified spermatozoa being unable then to induce $\mathrm{CI}$, (ii) the presence of nuclear restorer gene(s) suppressing CI defects, but not in all eggs or (iii) some specific interaction between the Wolbachia infecting both strains. In agreement with this latter hypothesis, Tunis males are able to induce complete CI (no larvae produced) when crossed with females from other strains than Slab (data not shown), whereas Slab females are entirely incompatible with MaClo males (no larvae produced; Table 1).

Knowledge of the CI phenotypes of embryos provides pivotal information for the understanding of the Wolbachia CI mechanism. Our data, showing the heterogeneity of phenotypes within CI embryos, have, therefore, given new insights into the relationships between $C$. pipiens and Wolbachia. The Wolbachia strains involved, the infection status of the female and also male factors have been implicated in CI. These results from C. pipiens contrast with observations in other insect species, suggesting a novel CI mechanism.

\section{Acknowledgements}

We are very grateful to $\mathrm{N}$ Pasteur and $\mathrm{M}$ Raymond for helpful comments on the manuscript; C Bernard, S Unal, C Berticat and A Berthomieu for technical assistance; V Durand for bibliographic help. This work was financed 
in part by APR PNETOX 2001 (Ministère de l'Aménagement et du Territoire) and APR 'Evaluation et réduction des risques liés à l'utilisation des pesticides' (Ministère de l'Ecologie et du Développement Durable). 2006.20 of the Institut des Sciences de l'Evolution de Montpellier (UMR CNRS 5554).

\section{References}

Ben Cheikh H, Ben Ali-Haouas Z, Marquine M, Pasteur N (1998). Resistance to organophosphorus and pyrethroid insecticides in Culex pipiens (Diptera: Culicidae) from Tunisia. J Med Entomol 35: 251-260.

Berticat C, Rousset F, Raymond M, Berthomieu A, Weill M (2002). High Wolbachia density in insecticide-resistant mosquitoes. Proc R Soc London Ser B 269: 1413-1416.

Bourguet D, Foncesca D, Vourch G, Dubois MP, Chandre F, Severini C et al (1998). The acetylcholinesterase gene ace: a diagnostic marker of the pipiens and quinquefasciatus forms of the Culex pipiens complex. J Am Mosquito Contr Assoc 14: 390-396.

Bressac C, Rousset F (1993). The reproductive incompatibility system in Drosophila simulans: DAPI-staining analysis of the Wolbachia symbionts in sperm cysts. J Invertebrate Pathol 61: 226-230.

Callaini G, Riparbelli MG, Giordano R, Dallai R (1996). Mitotic defects associated with cytoplasmic incompatibility in Drosophila simulans. J Invertebrate Pathol 67: 55-64.

Clements AN (1992). The Biology of Mosquitoes. Chapman and Hall: London.

Crawley MJ (1993). GLIM for Ecologists. Blackwell: Oxford.

Dallal GE, Wilkinson L (1986). An analytic approximation to the distribution of Lilliefors' test for normality. Am Statistician 40: 294-296.

Duron O, Fort P, Weill M (2006). Hyper variable prophage WO sequences describe an unexpected high number of Wolbachia variants in the mosquito Culex pipiens. Proc $R$ Soc London Ser B 273: 495-502.

Duron O, Lagnel J, Raymond M, Bourtzis K, Fort P, Weill M (2005). Transposable element polymorphism of Wolbachia in the mosquito Culex pipiens: evidence of genetic diversity, super-infection and recombination. Mol Ecol 14: 1561-1573.

Georghiou GP, Metcalf RL, Gidden FE (1966). Carbamateresistance in mosquitoes: selection of Culex pipiens fatigans Wied (=Culex quinquefasciatus) for resistance to Baygon. Bull World Health Organ 35: 691-708.

Guillemaud T, Pasteur N, Rousset F (1997). Contrasting levels of variability between cytoplasmic genomes and incompatibility types in the mosquito Culex pipiens. Proc $R$ Soc London Ser B 264: 245-251.

Halliday WR, Georghiou GP (1985). Linkage relationships of the knockdown resistance gene $(k d r)$ in larvae of Culex quinquefasciatus (Diptera: Culicidae). J Med Entomol 22: 572-576.

Jost E (1970a). Genetische Untersuchungen zur inkompatibilitat im Culex pipiens komplex. Theoret Appl Genet 40: 251-256.

Jost E (1970b). Untersuchungen zur inkompatibilitat in Culex pipiens komplex. Wilhem Roux Archiv Für Entwicklungsmechanik der Organismen 166: 173-188.

Jost E (1971). Meiosis in the male of Culex pipiens and Aedes albopictus and fertilization in the Culex pipiens complex. Can J Genet Cytol 13: 237-250.
Lassy CW, Karr TL (1996). Cytological analysis of fertilization and early embryonic development in incompatible crosses of Drosophila simulans. Mech Dev 57: 47-58.

Laven H (1967). Speciation and evolution in Culex pipiens. In: Wright J, Pal R (eds) Genetics of Insect Vectors of Disease. Elsevier: Amsterdam.

Malcolm CA, Bourguet D, Ascolillo A, Rooker SJ, Garvey CF, Hall LMC et al (1998). A sex-linked Ace gene, not linked to insensitive acetylcholinesterase-mediated insecticide resistance in Culex pipiens. Insect Mol Biol 7: 107-120.

Martinez-Torres D, Chevillon C, Brun-Barale A, Bergé JB, Pasteur N, Pauron D (1999). Voltage-dependent $\mathrm{Na}^{+}$channel in pyrethroid-resistant Culex pipiens L. mosquitoes. Pestic Sci 55: $1012-1020$.

Merçot H, Charlat S (2003). Wolbachia infections in Drosophila melanogaster and D. simulans: polymorphism and levels of cytoplasmic incompatibility. Genetica 120: 51-59.

Otsuka Y, Takaoka H (1997). Elimination of Wolbachia pipientis from Aedes albopictus. Med Entomol Zool 48: 257-260.

Portaro JK, Barr RA (1975). 'Curing' Wolbachia infections in Culex pipiens. J Med Entomol 12: 265.

R Development Core Team (2004). R: A Language and Environment for Statistical Computing. R Foundation for statistical computing: Vienna, Austria.

Rasgon JL, Scott TW (2003). Wolbachia and cytoplasmic incompatibility in the California Culex pipiens mosquito species complex: parameter estimates and infection dynamics in natural populations. Genetics 165: 2029-2038.

Raymond M, Magnin M, Pasteur N, Pasteur G, Sinègre G (1986). Cytoplasmic incompatibility in the mosquito Culex pipiens L. from southern France: implications for the selection and dispersal of insecticide resistance genes in natural populations. Genetica 70: 113-118.

Rogers SO, Bendich AJ (1988). Extraction of DNA from plant tissues. In: Gelvin SB, Schilperoort RA (eds) Plant Molecular Biology Manuel. Kluwer Academic Publishers: Boston. pp 1-10.

Rousset F, Raymond M (1991). Cytoplasmic incompatibility in insects: why sterilize females? Trends Ecol Evol 6: 54-57.

Stouthamer R, Breeuwer JAJ, Hurst GDD (1999). Wolbachia pipientis: microbial manipulator of arthropod reproduction. Annu Rev Microbiol 53: 71-102.

Tram U, Ferree PM, Sullivan W (2003). Identification of Wolbachia - host interacting factors through cytological analysis. Microbes Infect 5: 999-1011.

Weill M, Berticat C, Raymond M, Chevillon C (2000). Quantitative polymerase chain reaction to estimate the number of amplified esterase genes in insecticide-resistant mosquitoes. Anal Biochem 285: 267-270.

Weill M, Lutfalla G, Mogensen K, Chandre F, Berthomieu A, Berticat $C$ et al (2003). Insecticide resistance in mosquito vectors. Nature 423: 136-137.

Werren JH (1997). Biology of Wolbachia. Annu Rev Entomol 42: $587-609$.

Wright JD, Barr AR (1981). Wolbachia and the normal and incompatible eggs of Aedes polynesiensis. J Invertebrate Pathol 38: 409-418.

Xi L, Dean JL, Khoo C, Dobson SL (2005). Generation of a novel Wolbachia infection in Aedes albopictus (Asian tiger mosquito) via embryonic microinjection. Insect Biochem Mol Biol 35: 903-910. 